\title{
Tofacitinib shows promise in PsA trials
}

The findings of two phase III trials, Oral Psoriatic Arthritis Trial (OPAL) Broaden and OPAL Beyond, support

At 3 months, tofacitinib was superior to placebo with regard to both primary end points and may be toxic for some patients. Therefore, a drug with a different mechanism of action might work better," she continues.

"The unmet need in PsA is high [now] and [was] especially several years ago when very few treatment options were available," adds Philip Mease, corresponding author of the OPAL Broaden trial. "Basic science data supported the concept that tofacitinib would demonstrate efficacy via inhibition (directly or indirectly) of multiple cytokines that are involved in PsA," he says.

OPAL Broaden enrolled 422 patients with PsA who had failed to respond to previous treatment with a TNF inhibitor, and OPAL Beyond involved 395 patients with PsA who had not previously been treated with a TNF inhibitor. In both studies, patients were randomly assigned to receive either tofacitinib $5 \mathrm{mg}$ twice daily, tofacitinib $10 \mathrm{mg}$ twice daily or placebo for 3 months; the OPAL Broaden trial also included a group who received an active control, adalimumab $40 \mathrm{mg}$ every 2 weeks.

Notably, all patients enrolled in the studies were required to receive stable background treatment with a conventional synthetic DMARD, such as methotrexate, sulfasalazine or leflunomide. In addition, the placebo period was limited to 3 months, after which all patients in the placebo groups were switched to tofacitinib at either the $5 \mathrm{mg}$ or the $10 \mathrm{mg}$ twice daily dose. Each trial had a total duration of 6 months.

At 3 months, tofacitinib was superior to placebo with regard to both primary end points: the proportion of patients who achieved an ACR20 response (ACR criteria for $\geq 20 \%$ improvement) and change from baseline in the Health Assessment Questionnaire disability index (HAQ-DI) score.

"If approved, tofacitinib will expand the options available to patients with active PsA," says Mease. "It appears that the drug can be reasonably used [at] various points along the treatment path, including before biologics or after biologics have been tried," he adds. Further studies are needed to determine the long-term safety and efficacy of tofacitinib in PsA, and how it will be used alongside other available treatments.

Sarah Onuora

ORIGINAL ARTICLES Gladman, D. et al. Tofacitinib for psoriatic arthritis in patients with an inadequate response to TNF inhibitors. N. Engl. J. Med. 377, 1525-1536 (2017) | Mease, P. et al. Tofacitinib or adalimumab versus placebo for psoriatic arthritis. N. Engl.J. Med. 377, 1537-1550 (2017) 